\title{
Determining attitude and readiness of Medical students in Kerman University of Medical Sciences concerning acceptance of Decision Support Systems for Electronic Prescription in 2017
}

\section{Type of article: conference abstract}

\author{
Maryam Eslami Jahromi1, Fatemeh Tabatabaei 1, Reza Khajouei2* \\ 1: Master Student of Health Information Technology, Faculty of Management and \\ Medical Information Sciences, Kerman University of Medical Sciences, Kerman, Iran. \\ 2: Associate Professor, Medical Informatics Research Center, Institute for Future Studies \\ in Health, Kerman. University of Medical Sciences, Kerman, Iran. \\ *Tel: +98.3431325406, Fax: +98.3431325221, E-mail: r.khajouei@yahoo.com
}

\begin{abstract}
Introduction: Clinical Decision Support Systems (CDSS) are any computer program designed to assist health care professionals in making clinical decisions, especially at the moment of action. These information systems are flexible and interactive, helping people by making use of models and rules of a comprehensive database, or on the basis of clinical guidelines, to make applicable decisions, especially solving difficult problems with common scientific methods. Therefore, these systems support complex medical decisions, increase their effectiveness, and can lead to a reduction in the types of medical errors, such as medication errors. However, knowledge and attitude of the future physicians in the country about these systems is unclear. The purpose of this study was Determining attitude and readiness of Medical students concerning acceptance of Decision Support Systems for Electronic Prescription.

Methods: This research is descriptive-analytic. The research population included general medical students of Kerman University of Medical Sciences in 2017. Out of these students, a sample of 226 people was selected by proportional sampling. Data collection was done using a standard questionnaire. The questionnaire was first translated into Persian and then its validity was confirmed by three medical informatics specialists. Data analysis was done descriptivelyanalytically using SPSS 19.

Results: The findings of this study showed that $17 \%$ of students $(n=36)$ knew about this system and how to use it, and $83 \%$ of them $(n=177)$ were willing to learn and use this system to practice their profession in the future. More than half of them $(n=121)$ believed that using this system would Reduce errors in prescribing. $80 \%$ of them $(n=170)$ lacked a strategic plan to implement a decision support system for electronic prescription and a lack of standard and acceptable software, and about $60 \%(\mathrm{n}=128)$ lack of financial incentives and lack of sufficient evidence Concerned about the usefulness of using it as a potential obstacle to the implementation of this system. There was no significant difference between the age, sex and term of the students with their familiarity with this system $(\mathrm{P}>0.05)$.

Conclusion: The results of this study showed that while many students lack sufficient knowledge about the clinical decision support system, they are interested in learning and using it, especially in the field of prescribing drugs. This result indicates that the system is likely to be admitted if it is implemented and educated to medical students and doctors.
\end{abstract}

KEYWORDS: Technology Acceptance, Clinical Decision Support System, Medical Students.

\section{Declaration of conflicts}

This abstract is selected from the First International Congress of Diseases and Health Outcomes Registry and First National Congress of Medical Informatics, 14-17 February 2017, Mashhad, Iran 
Medical Technologies Journal, Volume: 1, Issue: 4, October-December 2017, Pages:76-136. Doi : https://doi.org/10.26415/2572-004X-vol1iss4

\section{Authors' biography}

No biography.

\section{References}

No references. 